Research Article

\title{
Hybrid Structures Applied to Ideals in BCI-Algebras
}

\author{
G. Muhiuddin (D), ${ }^{1}$ D. Al-Kadi, ${ }^{2}$ and A. Mahboob ${ }^{3}$ \\ ${ }^{1}$ Department of Mathematics, University of Tabuk, Tabuk 71491, Saudi Arabia \\ ${ }^{2}$ Department of Mathematics and Statistic, College of Science, Taif University, P.O. Box 11099, Taif 21944, Saudi Arabia \\ ${ }^{3}$ Madanapalle Institute of Technology \& Science, Madanapalle-517325, India
}

Correspondence should be addressed to G. Muhiuddin; chishtygm@gmail.com

Received 8 July 2020; Revised 18 September 2020; Accepted 4 November 2020; Published 26 November 2020

Academic Editor: Ali Jaballah

Copyright (C) 2020 G. Muhiuddin et al. This is an open access article distributed under the Creative Commons Attribution License, which permits unrestricted use, distribution, and reproduction in any medium, provided the original work is properly cited.

In this paper, the notion of hybrid structure is applied to the ideal theory in BCI-algebras. In fact, we introduce the notions of hybrid $p$-ideal, hybrid $h$-ideal, and hybrid $a$-ideal in BCI-algebras and investigate their related properties. Furthermore, we show that every hybrid $p$-ideal (or $h$-ideal or $a$-ideal) is a hybrid ideal in a BCI-algebra but converse need not be true in general and in support, and we exhibit counter examples for each case. Moreover, we consider characterizations of hybrid $p$-ideal, hybrid $h$-ideal, and hybrid $a$-ideal in BCI-algebras.

\section{Introduction}

Imai and Iséki $[1,2]$ initiated the study of "BCK/BCI-algebras" in 1966 as a generalization of the notions of settheoretical difference and propositional calculus. A great deal of literature has been developed on the theory of (BCK/BCI)-algebras since then, in particular, more focus has been placed on the "ideal theory" of BCK/BCI-algebras. In (BCK/BCI)-algebras, different kinds of ideals on different aspects have been studied (see, for example, [3-6]).

Fuzzy sets, introduced by Zadeh [7], deal with potential uncertainties, related to the imprecision of states, perceptions, and preferences. Molodtsov [8] proposed the concept of a "soft set" as a new mathematical framework for dealing with uncertainties, free of the difficulties that have disrupted normal theoretical approaches. Molodtsov pointed out a number of ways to develop soft sets. Molodtsov applied soft set theory in a variety of ways, such as smoothness of functions, game theory, operational research, Riemann integration, Perron integration, probability, and measurement theory (see [8-10]). Algebraic structures such as (BCK/BCI)-algebra [11], $d$-algebras [12], group [13], semigroup [14], ring [15], semiring [16], and decisionmaking $[17,18]$ are theoretically applied by soft set theory. Muhiuddin et al. (see, for example, [19-23]) investigated the fuzzy set theoretical approach to the (BCK/BCI)-algebras on various aspects. Further concepts related to this analysis in different fields have also been studied in [24-34].

In a system of parameters, Jun et al. [35] proposed the concept of "hybrid structure" over the initial universe set by merging fuzzy sets and soft sets. The idea of a hybrid subalgebra, a hybrid field, and a hybrid linear space was introduced with this notion. The hybrid structure theory and its applications to (BCK/BCI)-algebras and semigroups have recently been studied in (see [36-40] and references).

The objective of this paper is to introduce the notions of hybrid $p$-ideal, hybrid $h$-ideal, and hybrid $a$-ideal in (BCI)-algebras and investigate their related properties. Furthermore, we show that hybrid ( $p$-ideal, $h$-ideal, and $a$ ideal) are all hybrid ideals, but converse need not be true in general and in support, and we exhibit counter examples. Also, we provide conditions for a hybrid $p$-ideal (or hybrid $h$-ideal or hybrid $a$-ideal) to be a hybrid ideal.

\section{Preliminaries}

An algebra $Z=(Z ; *, 0)$ of type $(2,0)$ is a $(\mathrm{BCI})$-algebra if it satisfies for all $s, t, r \in Z$ : 


$$
\begin{array}{r}
\left(K_{1}\right)((s * t) *(s * r)) *(r * t)=0 \\
\left(K_{2}\right)(s *(s * t)) * t=0, \\
\left(K_{3}\right) s * s=0, \\
\left(K_{4}\right) s * t=0 \text { and } t * s=0 \Rightarrow s=t
\end{array}
$$

If a (BCI)-algebra $Z$ satisfies

$$
\left(K_{5}\right) 0 * s=0,
$$

then $Z$ is a $B C K$ - algebra.

Any (BCK/BCI)-algebra $Z$ satisfies the following conditions:

$$
\begin{aligned}
\left(P_{1}\right) s * 0 & =s, \\
\left(P_{2}\right)(s * t) * r & =(s * r) * t, \\
\left(P_{3}\right) s & \leq t \Rightarrow s * r \leq t * r \text { and } r * t \leq r * s, \\
\left(P_{4}\right)(s * r) *(t * r) & \leq(s * t),
\end{aligned}
$$

where $s \leq t$ if and only if $s * t=0$. Note that $(Z, \leq)$ is a partially ordered set.

Any (BCI)-algebra $Z$ satisfies the following conditions $[41,42]$ :

$$
\begin{aligned}
\left(P_{5}\right) 0 *(0 *(s * t)) & =0 *(t * s), \\
\left(P_{6}\right) 0 *(s * t) & =(0 * s) *(0 * t), \\
\left(P_{7}\right) s *(s *(s * t)) & =s * t \\
\left(P_{8}\right) 0 *(0 *(s * t)) & =(0 * t) *(0 * s) .
\end{aligned}
$$

For more details on (BCK)-algebras and (BCI)-algebras, we refer the readers to [43-45].

A subset $(\varnothing \neq) T$ of a $(\mathrm{BCK} / \mathrm{BCI})$-algebra $Z$ is called a subalgebra if $t * s \in T \forall t, s \in T$ and is called an ideal of $Z$ if $0 \in T$ and $\forall t, s \in Z, t * s \in T, s \in T$ implies $t \in T$. Furthermore, a subset $(\varnothing \neq) T$ of BCI-algebra $Z$ is called $p$-ideal (resp. $q$-ideal and $a$-ideal) if $0 \in T$ and $\forall t, s, r \in Z,((t * r) *(s * r)) \in T, s \in T$ implies $t \in T$ (resp. $(t *(s * r)) \in T, s \in T \quad$ implies $\quad t * r \in T$, and $((t * r) *(0 * s)) \in T, r \in T$ implies $s * t \in T)$.

Definition 1 (see [35]). For a set of parameters $Z$, an initial universe set $U$, a power set of the initial set $P(U)$, and the unit interval $I$, a hybrid structure (briefly, HS) in $Z$ over $U$ is defined to be a mapping $\tilde{g}_{\delta}:=(\widetilde{g} ; t \tilde{\delta}): Z \longrightarrow P(U) \times I ; z \mapsto(\widetilde{g}(z) t ; n \tilde{\delta} q(z))$, where $\widetilde{g}: Z \longrightarrow P(U)$ and $\widetilde{\delta}: Z \longrightarrow I$ are mappings.

Definition 2 (see [35]). Let $Z$ be a (BCK/BCI)-algebra. For a HS $\tilde{g}_{\delta}$ in $Z$ over $U, \widetilde{g}_{\delta}$ is said to be a hybrid subalgebra of $Z$ if the following statements are valid:

$$
(\forall s, t \in Z) \quad\left(\begin{array}{c}
\widetilde{g}(s * t) \supseteq \widetilde{g}(s) \cap \widetilde{g}(t), \\
\widetilde{\delta}(s * t) \leq \vee\{\widetilde{\delta}(s), \widetilde{\delta}(t)\}
\end{array}\right) .
$$

Definition 3 (see [39]). A HS $\tilde{g}_{\delta}$ in $Z$ over $U$ is said to be a hybrid ideal of $Z$ over $U$ if

$$
\begin{array}{r}
\left(\mathrm{HI}_{1}\right)(\forall s \in Z) \quad\left(\begin{array}{c}
\tilde{g}(0) \supseteq \tilde{g}(s), \\
\tilde{\delta}(0) \leq \tilde{\delta}(s)
\end{array}\right), \\
\left(\mathrm{HI}_{2}\right)(\forall s, t \in Z) \quad\left(\begin{array}{c}
\tilde{g}(s) \supseteq \tilde{g}(s * t) \cap \tilde{g}(t), \\
\tilde{\delta}(s) \leq \mathrm{V}\{\tilde{\delta}(s * t), \widetilde{\delta}(t)\}
\end{array}\right) .
\end{array}
$$

Proposition 1 (see [39]). Let $\tilde{g}_{\delta}=(\widetilde{g}, t \widetilde{\delta})$ be a hybrid ideal of $Z$. If the inequality $s \leq t$ holds in $Z$, then $\widetilde{g}(s) \supseteq \widetilde{g}(t)$ and $\widetilde{\delta}(s) \leq \widetilde{\delta}(t)$.

\section{Hybrid Ideals in BCI-Algebras}

Throughout the following sections $Z$ denotes BCI-algebra unless stated otherwise.

Definition 4. A HS $\tilde{g}_{\delta}$ in $Z$ over $U$ is said to be a hybrid $p$-ideal of $Z$ over $U$ if it satisfies $\left(\mathrm{HI}_{1}\right)$ and $\left(\mathrm{HI}_{3}\right)(\forall s, t, r \in Z)(\widetilde{g}(s) \supseteq \widetilde{g}((s * r) *(t * r)) \cap \widetilde{g}(t), \widetilde{\delta}(s) \leq$ $\vee\{\widetilde{\delta}((s * r) *(t * r)), \widetilde{\delta}(t)\})$.

Example 1. Let $U=\left\{\gamma_{1}, \gamma_{2}, \gamma_{3}, \gamma_{4}\right\}$ be the initial universe set. On a set of parameters $Z=\{f, s, n, t\}$, we define the operation $*$ in Table 1 .

Then, $(Z, *, f)$ is a (BCI)-algebra. Let $\tilde{g}_{\delta}$ be a HS in $Z$ over $U$ which is given by Table 2 .

By routine verification $\tilde{g}_{\delta}$ is a hybrid $p$-ideal of $Z$ over $U$.

Theorem 1. For any (BCI)-algebra, every hybrid p-ideal is a hybrid ideal.

Proof. Suppose that $\tilde{g}_{\delta}$ is a hybrid $p$-ideal of $Z$ over $U$. Since $s * 0=s$ for all $s \in Z$, we have

$$
\begin{aligned}
\tilde{g}(s) & \supseteq \widetilde{g}((s * 0) *(t * 0)) \cap \tilde{g}(t) \\
& \supseteq \widetilde{g}(s * t) \cap \widetilde{g}(t), \\
\widetilde{\delta}(s) & \leq \cup\{\widetilde{\delta}((s * 0) *(t * 0)), \widetilde{\delta}(t)\}, \\
& \leq \cup\{\widetilde{\delta}(s * t), \widetilde{\delta}(t)\} .
\end{aligned}
$$

for all $s, t \in Z$. Hence, $\tilde{g}_{\delta}$ is a hybrid ideal of $Z$ over $U$.

As shown in the following example, the converse of Theorem 1 is not generally valid.

Example 2. Let $U=\left\{\gamma_{1}, \gamma_{2}, \gamma_{3}, \gamma_{4}, \gamma_{5}\right\}$ be the initial universe set and $Z=\{f, s, n, t, e\}$ be the set of parameters. We define the binary operation $*$ on $Z$ by Table 3 .

Then, $(Z, *, f)$ is a $(\mathrm{BCI})$-algebra. Now, define a HS $\tilde{g}_{\delta}$ in $Z$ over $U$ which is given by Table 4 .

It is easy to check that $\tilde{g}_{\delta}$ is a hybrid ideal of $Z$ over $U$ but not hybrid $p$-ideal as

$$
\begin{aligned}
\tilde{g}(n) & =\left\{\gamma_{2}, \gamma_{4}\right\} \supseteq\left\{\gamma_{1}, \gamma_{2}, \gamma_{3}, \gamma_{4}\right\}=\tilde{g}((n * n) *(s * n)) \cap \tilde{g}(s) \\
& =\widetilde{g}(f * s) \cap \tilde{g}(s)=\widetilde{g}(f) \cap \tilde{g}(s) .
\end{aligned}
$$

Theorem 2. If $\tilde{g}_{\delta}$ is a hybrid p-ideal of $Z$ over $U$, then

$$
\begin{gathered}
\widetilde{g}(s) \supseteq \widetilde{g}(0 *(0 * s)), \\
\widetilde{\delta}(s) \leq \widetilde{\delta}(0 *(0 * s)),
\end{gathered}
$$


TABLE 1: Cayley table of the binary operation*.

\begin{tabular}{lllll}
\hline$*$ & $f$ & $s$ & $n$ & $t$ \\
\hline$f$ & $f$ & $s$ & $n$ & $t$ \\
$s$ & $s$ & $f$ & $t$ & $n$ \\
$n$ & $n$ & $t$ & $f$ & $s$ \\
$t$ & $t$ & $n$ & $s$ & $f$ \\
\hline
\end{tabular}

TABLE 2: Table representation of hybrid structure $\tilde{g}_{\delta}$.

\begin{tabular}{lcc}
\hline$Z$ & $\widetilde{g}$ & $\tilde{\delta}$ \\
\hline$f$ & $U$ & 0.3 \\
$s$ & $\left\{\gamma_{1}, \gamma_{2}, \gamma_{3}\right\}$ & 0.4 \\
$n$ & $\left\{\gamma_{1}, \gamma_{2}\right\}$ & 0.5 \\
$t$ & $\left\{\gamma_{1}, \gamma_{2}\right\}$ & 0.5 \\
\hline
\end{tabular}

TABLE 3: Cayley table of the binary operation*.

\begin{tabular}{llllll}
\hline$*$ & $f$ & $s$ & $n$ & $t$ & $e$ \\
\hline$f$ & $f$ & $f$ & $f$ & $t$ & $t$ \\
$s$ & $s$ & $f$ & $s$ & $e$ & $t$ \\
$n$ & $n$ & $n$ & $f$ & $t$ & $t$ \\
$t$ & $t$ & $t$ & $t$ & $f$ & $f$ \\
$e$ & $e$ & $t$ & $e$ & $s$ & $f$ \\
\hline
\end{tabular}

TABLE 4: Table representation of hybrid structure $\tilde{g}_{\delta}$.

\begin{tabular}{lcc}
\hline$Z$ & $\widetilde{g}$ & $\delta$ \\
\hline$f$ & $U$ & 0.4 \\
$s$ & $\left\{\gamma_{1}, \gamma_{2}, \gamma_{3}, \gamma_{4}\right\}$ & 0.8 \\
$n$ & $\left\{\gamma_{2}, \gamma_{4}\right\}$ & 0.5 \\
$t$ & $\left\{\gamma_{2}, \gamma_{4}\right\}$ & 0.7 \\
$e$ & $\left\{\gamma_{2}, \gamma_{4}\right\}$ & 0.8 \\
\hline
\end{tabular}

for all $s \in Z$.

Proof. Let $\tilde{g}_{\delta}$ be a hybrid $p$-ideal of $Z$ over $U$. Then,

$$
\begin{aligned}
& \widetilde{g}(s) \supseteq \widetilde{g}((s * r) *(t * r)) \cap \widetilde{g}(t), \\
& \widetilde{\delta}(s) \leq \vee\{\widetilde{\delta}((s * r) *(t * r)), \widetilde{\delta}(t)\},
\end{aligned}
$$

for all $s, t, r \in Z$. Substituting $s$ for $r$ and 0 for $t$ in (10), then

$$
\begin{aligned}
\widetilde{g}(s) & \supseteq \widetilde{g}(\widetilde{g}((s * s) *(0 * s)), \widetilde{g}(0)) \\
& =\widetilde{g}(0 *(0 * s)) \cap \widetilde{g}(0) \\
& =\widetilde{g}(0 *(0 * s)), \\
\widetilde{\delta}(s) & \leq \cup\{\widetilde{\delta}((s * s) *(0 * s)), \widetilde{\delta}(0)\} \\
& =\cup\{\widetilde{\delta}(0 *(0 * s)), \widetilde{\delta}(0)\} \\
& =\widetilde{\delta}(0 *(0 * s)) .
\end{aligned}
$$

Theorem 3. If $\tilde{g}_{\delta}$ is a hybrid p-ideal of $Z$ over $U$, then

$$
\begin{aligned}
& \tilde{g}(s)=\widetilde{g}(0 *(0 * s)), \\
& \widetilde{\delta}(s)=\widetilde{\delta}(0 *(0 * s)),
\end{aligned}
$$

for all $s \in Z$.
Proof. By $\left(K_{2}\right),(0 *(0 * s)) \leq s$. Therefore, by Proposition 1 ,

$$
\begin{gathered}
\widetilde{g}(0 *(0 * s)) \supseteq \widetilde{g}(s), \\
\widetilde{\delta}(0 *(0 * s)) \leq \widetilde{\delta}(s) .
\end{gathered}
$$

Thus, by using (9), we have

$$
\begin{aligned}
\widetilde{g}(s) & =\widetilde{g}(0 *(0 * s)), \\
\widetilde{\delta}(s) & =\widetilde{\delta}(0 *(0 * s)) .
\end{aligned}
$$

Theorem 4. If $\tilde{g}_{\delta}$ is a hybrid p-ideal of $Z$ over $U$, then

$$
\begin{aligned}
& \widetilde{g}((s * r) *(t * r)) \supseteq \widetilde{g}(s * t), \\
& \widetilde{\delta}((s * r) *(t * r)) \leq \widetilde{\delta}(s * t),
\end{aligned}
$$

for all $s, t, r \in Z$.

Proof. Let $\tilde{g}_{\delta}$ be a hybrid $p$-ideal of $Z$ over $U$. Note that $((s * r) *(t * r) \leq s * t)$ holds in $Z$. It implies that $((s * r) *(t * r)) *(s * t)=0$. Since $\tilde{g}_{\delta}$ is a hybrid ideal of $Z$ over $U$, we have

$$
\begin{aligned}
\widetilde{g}((s * r) *(t * r)) & \supseteq \widetilde{g}(((s * r) *(t * r)) *(s * t)) \cap \widetilde{g}(s * t) \\
& =\widetilde{g}(0) \cap \widetilde{g}(s * t) \\
& =\widetilde{g}(s * t), \\
\widetilde{\delta}((s * r) *(t * r)) & \leq \cup\{\widetilde{\delta}(((s * r) *(t * r)) \\
& *(s * t)), \widetilde{\delta}(s * t)\} \\
& =\cup\{\widetilde{\delta}(0), \widetilde{\delta}(s * t)\} \\
& =\widetilde{\delta}(s * t),
\end{aligned}
$$

for all $s, t, r \in Z$.

The following theorem gives a condition for a hybrid ideal to be a hybrid $p$-ideal.

Theorem 5. Let $\tilde{g}_{\delta}$ be a hybrid ideal of $Z$ over $U$ satisfying the following condition:

$$
\begin{aligned}
& \widetilde{g}(s * t) \supseteq \widetilde{g}((s * r) *(t * r)), \\
& \widetilde{\delta}(s * t) \leq \widetilde{\delta}((s * r) *(t * r)),
\end{aligned}
$$

for all $s, t, r \in Z$. Then, $\tilde{g}_{\delta}$ is a hybrid $p$-ideal of $Z$ over $U$.

Proof. Suppose that $\tilde{g}_{\delta}$ is a hybrid ideal of $Z$ satisfying (17). Then,

$$
\begin{aligned}
\tilde{g}(s) & \supseteq \widetilde{g}(s * t) \cap \tilde{g}(t) \\
& \supseteq \widetilde{g}((s * r) *(t * r)) \cap \widetilde{g}(t), \\
\widetilde{\delta}(s) & \leq \cup\{\widetilde{\delta}(s * t), \widetilde{\delta}(t)\} \\
& \leq \cup\{\widetilde{\delta}((s * r) *(t * r)), \widetilde{\delta}(t)\},
\end{aligned}
$$

for all $s, t, r \in Z$, as required. 
Definition 5. A HS $\tilde{g}_{\delta}$ in $Z$ over $U$ is said to be a hybrid $h$ ideal of $Z$ over $U$ if it satisfies $\left(\mathrm{HI}_{1}\right)$ and $\left(\mathrm{HI}_{4}\right)$ $(\forall s, t, r \in Z) \quad\left(\begin{array}{c}\widetilde{g}(s * r) \supseteq \widetilde{g}(s *(t * r)) \cap \widetilde{g}(t), \\ \widetilde{\delta}(s * r) \leq \cup\{\tilde{\delta}(s *(t * r)), \widetilde{\delta}(t)\}\end{array}\right)$.

Example 3. Let $U=\left\{\gamma_{1}, \gamma_{2}, \gamma_{3}, \gamma_{4}, \gamma_{5}\right\}$ be the initial universe set. As a set of parameters, we consider $Z=\{f, s, n, t, e\}$. The operation $*$ is defined on $Z$ by Table 5 .

Then, $(Z, *, f)$ is a (BCI)-algebra. Define a $\mathrm{HS} \tilde{g}_{\delta}$ in $Z$ over $U$ by the following Table 6 .

It can be easily checked that $\tilde{g}_{\delta}$ is a hybrid h-ideal of $Z$ over $U$.

Theorem 6. For any (BCI)-algebra, every hybrid h-ideal is a hybrid ideal.

Proof. Proof. Let $\tilde{g}_{\delta}$ be a hybrid $h$-ideal of $Z$ over $U$. Since $s * 0=s$ for all $s \in Z$, we have

$$
\begin{array}{r}
\tilde{g}(s * 0) \supseteq \tilde{g}(s *(t * 0)) \cap \tilde{g}(t) \\
\tilde{g}(s) \supseteq \widetilde{g}(s * t) \cap \widetilde{g}(t) \\
\text { and } \\
\widetilde{\delta}(s * 0) \leq \cup\{\widetilde{\delta}(s *(t * 0)), \widetilde{\delta}(t)\} \\
\widetilde{\delta}(s) \leq \cup\{\widetilde{\delta}(s * t), \widetilde{\delta}(t)\},
\end{array}
$$

for all $s, t \in Z$. Hence, $\tilde{g}_{\delta}$ is a hybrid ideal of $Z$ over $U$.

In general, the converse of Theorem 6 is not valid, as the following example shows.

Example 4. Let $U=\left\{\gamma_{1}, \gamma_{2}, \gamma_{3}, \gamma_{4}, \gamma_{5}\right\}$ be the initial universe set. For a set of parameters $Z=\{p, s, t, w, q\}$, we define a binary operation by the Cayley table in Table 7 .

Then, $(Z, *, p)$ is a $(\mathrm{BCI})$-algebra. Define a $\mathrm{HS} \tilde{g}_{\delta}$ in $Z$ over $U$ by Table 8 .

It is routine to verify that $\tilde{g}_{\delta}$ is a hybrid ideal but not hybrid $h$-ideal since
TABLE 5: Cayley table of the binary operation*.

\begin{tabular}{llllll}
\hline$*$ & $f$ & $s$ & $n$ & $t$ & $e$ \\
\hline$f$ & $f$ & $f$ & $f$ & $t$ & $t$ \\
$s$ & $s$ & $f$ & $s$ & $e$ & $t$ \\
$n$ & $n$ & $n$ & $f$ & $t$ & $t$ \\
$t$ & $t$ & $t$ & $t$ & $f$ & $f$ \\
$e$ & $e$ & $t$ & $e$ & $s$ & $f$ \\
\hline
\end{tabular}

TABLE 6: Table representation of hybrid structure $\tilde{g}_{\delta}$.

\begin{tabular}{lcc}
\hline$Z$ & $\widetilde{g}$ & $\tilde{\delta}$ \\
\hline$f$ & $U$ & 0.1 \\
$s$ & $\left\{\gamma_{2}, \gamma_{4}\right\}$ & 0.7 \\
$n$ & $\left\{\gamma_{1}, \gamma_{2}, \gamma_{3}, \gamma_{4}\right\}$ & 0.3 \\
$t$ & $\left\{\gamma_{2}, \gamma_{3}, \gamma_{4}\right\}$ & 0.5 \\
$e$ & $\left\{\gamma_{2}, \gamma_{4}\right\}$ & 0.7 \\
\hline
\end{tabular}

TABLE 7: Cayley table of the binary operation*.

\begin{tabular}{llllll}
\hline$*$ & $p$ & $s$ & $t$ & $w$ & $q$ \\
\hline$p$ & $p$ & $p$ & $q$ & $w$ & $t$ \\
$s$ & $s$ & $p$ & $q$ & $w$ & $t$ \\
$t$ & $t$ & $t$ & $p$ & $q$ & $w$ \\
$w$ & $w$ & $w$ & $t$ & $p$ & $q$ \\
$q$ & $q$ & $q$ & $w$ & $t$ & $p$ \\
\hline
\end{tabular}

TABLE 8: Table representation of hybrid structure $\tilde{g}_{\delta}$.

\begin{tabular}{lrc}
\hline$Z$ & $\tilde{g}$ & $\tilde{\delta}$ \\
\hline$p$ & $U$ & 0.2 \\
$s$ & $\left\{\gamma_{1}, \gamma_{2}, \gamma_{3}, \gamma_{4}\right\}$ & 0.3 \\
$t$ & $\left\{\gamma_{2}, \gamma_{3}\right\}$ & 0.4 \\
$w$ & $\left\{\gamma_{2}, \gamma_{3}\right\}$ & 0.4 \\
$q$ & $\left\{\gamma_{2}, \gamma_{3}\right\}$ & 0.4 \\
\hline
\end{tabular}

$$
\begin{aligned}
& \tilde{g}(q * t)=\tilde{g}(w)=\left\{\gamma_{2},\right. \\
& \tilde{\delta}(q * t)=\widetilde{\delta}(w)=0.4 \\
& \text { et } \tilde{g}_{\delta} \text { be a hybrid h-ide } \\
& \widetilde{g}(0 * s) \supseteq \widetilde{g}(s), \\
& \widetilde{\delta}(0 * s) \leq \widetilde{\delta}(s),
\end{aligned}
$$

for all $s \in Z$.

Proof. Let $\tilde{g}_{\delta}$ be a hybrid $h$-ideal of $Z$ over $U$. Then,

$$
\begin{array}{r}
\widetilde{g}(s * r) \supseteq \widetilde{g}(s *(t * r)) \cap \widetilde{g}(t), \\
\widetilde{\delta}(s * r) \leq \cup\{\widetilde{\delta}(s *(t * r)), \widetilde{\delta}(t)\} .
\end{array}
$$

Substituting $s$ by $0, r$ by $s$, and $t$ by $s$, we have
Definition 6. A HS $\tilde{g}_{\delta}$ in $Z$ over $U$ is said to be a hybrid a-ideal of $Z$ over $U$ if it satisfies $\left(\mathrm{HI}_{1}\right)$ and $\left(\mathrm{HI}_{5}\right)$ $(\forall s, t, r \in Z) \quad\left(\begin{array}{c}\tilde{g}(t * s) \supseteq \widetilde{g}((s * r) *(0 * t)) \cap \tilde{g}(r), \\ \widetilde{\delta}(t * s) \leq \cup\{\widetilde{\delta}((s * r) *(0 * t)), \widetilde{\delta}(r)\} .\end{array}\right)$.

Example 5. Consider $\tilde{g}_{\delta}$ represented in Example 1. It is routine to verify that $\tilde{g}_{\delta}$ is a hybrid $a$-ideal of $Z$ over $U$. 
Theorem 8. Every hybrid a-ideal is both a hybrid subalgebra and a hybrid ideal of $Z$ over $U$.

Proof. Let $\tilde{g}_{\delta}$ be a hybrid a-ideal of $Z$ over $U$. By substituting $t=r=0$ in $\left(\mathrm{HI}_{5}\right)$ and using $\left(K_{3}\right)$ and $\left(P_{1}\right)$, we obtain

$$
\begin{array}{r}
\widetilde{g}(0 * s) \supseteq \widetilde{g}((s * 0) *(0 * 0)) \cap \widetilde{g}(0)=\widetilde{g}(s * 0) \cap \widetilde{g}(0)=\widetilde{g}(s), \\
\widetilde{\delta}(0 * s) \leq \cup\{\widetilde{\delta}((s * 0) *(0 * 0)), \widetilde{\delta}(0)\}=\widetilde{\delta}(s),
\end{array}
$$

for all $s, t, r \in Z$.

Again setting $s=r=0$ in $\left(\mathrm{HI}_{5}\right)$, using $\left(P_{1}\right),\left(K_{3}\right),\left(\mathrm{HI}_{1}\right)$, and above equations, we obtain

$$
\begin{aligned}
\widetilde{g}(t * 0) \supseteq \widetilde{g}((0 * 0) *(0 * t)) \cap \widetilde{g}(0) \\
=\widetilde{g}(0 *(0 * t)) \cap \widetilde{g}(0)=\widetilde{g}(0 *(0 * t)) \supseteq \widetilde{g}(0 * t),
\end{aligned}
$$

which implies $\widetilde{g}(t) \supseteq \widetilde{g}(0 * t)$

$$
\begin{aligned}
\widetilde{\delta}(t * 0) & \leq \cup\{\widetilde{\delta}(0 *(0 * t)), \widetilde{\delta}(0)\} \\
& =\widetilde{\delta}(0 *(0 * t)) \leq \widetilde{\delta}(0 * t),
\end{aligned}
$$

which implies $\widetilde{\delta}(t) \leq \widetilde{\delta}(0 * t)$ for all $t \in Z$. Then, from $\left(\mathrm{HI}_{5}\right)$ and $\left(P_{1}\right)$, it implies that

$$
\begin{aligned}
\widetilde{g}(t) & \supseteq \widetilde{\mathcal{g}}(0 * t) \supseteq \widetilde{g}((t * r) *(0 * 0)) \cap \tilde{g}(r) \\
& =\widetilde{g}(t * r) \cap \widetilde{g}(r), \\
\widetilde{\delta}(t) & \leq \widetilde{\delta}(0 * t) \leq \cup\{\widetilde{\delta}((t * r) *(0 * 0)), \widetilde{\delta}(r)\} \\
& \leq \cup\{\widetilde{\delta}(t * r), \widetilde{\delta}(r)\},
\end{aligned}
$$

for all $t, r \in Z$. Hence, $\tilde{g}_{\delta}$ is a hybrid ideal of $Z$ over $U$.

Now, for any $t, r \in Z$ and using above equations, we have

$$
\begin{aligned}
\widetilde{g}(t * r) & \supseteq \widetilde{g}((t * r) * t) \cap \widetilde{g}(t) \\
& =\widetilde{g}(0 * r) \cap \widetilde{g}(t) \supseteq \widetilde{g}(t) \cap \widetilde{g}(r), \\
\widetilde{\delta}(t * r) & \leq \cup\{\widetilde{\delta}((t * r) * t), \widetilde{\delta}(t)\} \\
& =\cup\{\widetilde{\delta}(0 * r), \widetilde{\delta}(t)\} \leq \cup\{\widetilde{\delta}(t), \widetilde{\delta}(r)\} .
\end{aligned}
$$

Therefore, $\tilde{g}_{\delta}$ is a hybrid subalgebra of $Z$ over $U$. The converse of Theorem 8 is not generally valid as seen in the following example.
TABle 9: Cayley table of the binary operation*.

\begin{tabular}{llll}
\hline$*$ & $p$ & $s$ & $t$ \\
\hline$p$ & $p$ & $p$ & $p$ \\
$s$ & $s$ & $p$ & $s$ \\
$t$ & $t$ & $t$ & $p$ \\
\hline
\end{tabular}

TABLE 10: Table representation of hybrid structure $\tilde{g}_{\delta}$.

\begin{tabular}{lcc}
\hline$Z$ & $\tilde{g}$ & $\tilde{\delta}$ \\
\hline$p$ & $U$ & 0.6 \\
$s$ & $\left\{\gamma_{1}, \gamma_{2}, \gamma_{3}\right\}$ & 0.7 \\
$t$ & $\left\{\gamma_{1}, \gamma_{2}, \gamma_{3}\right\}$ & 0.7 \\
\hline
\end{tabular}

Example 6. Let $U=\left\{\gamma_{1}, \gamma_{2}, \gamma_{3}, \gamma_{4}, \gamma_{5}\right\}$ be the initial universe set. For a set of parameters $Z=\{p, s, t\}$, we define a binary operation by the Cayley table in Table 9.

Then, $(Z, *, p)$ is a $(\mathrm{BCI})$-algebra. Let $\tilde{g}_{\delta}$ be a HS in $Z$ over $U$ which is given by Table 10 .

Then, it directs to show that $\tilde{g}_{\delta}$ is a hybrid subalgebra and hybrid ideal but not hybrid $a$-ideal as

$$
\begin{aligned}
\widetilde{g}(s * p) & =\widetilde{g}(s)=\left\{\gamma_{1}, \gamma_{2}, \gamma_{3}\right\} \supseteq U \\
& =\widetilde{g}((p * p) *(p * s)) \cap \tilde{g}(p) \\
& =\widetilde{g}(p * p) \cap \widetilde{g}(p)=\widetilde{g}(p) \cap \tilde{g}(p), \\
\widetilde{\delta}(s * p) & =\widetilde{\delta}(s) \\
& =0.7 \leq \cup\{\widetilde{\delta}((p * p) *(p * s)), \widetilde{\delta}(p)\} \\
& =\cup\{\widetilde{\delta}(p * p), \widetilde{\delta}(p)\}=\cup\{\widetilde{\delta}(p), \widetilde{\delta}(p)\} \\
& =0.6 .
\end{aligned}
$$

Theorem 9. If $\tilde{g}_{\delta}$ is a hybrid a-ideal of $Z$ over $U$, then $\widetilde{g}((s * r) *(0 * t)) \supseteq \widetilde{g}(s *(r * t))$ and $\tilde{\delta}((s * r) *(0 * t)) \leq \widetilde{\delta}(s *(r * t))$ for all $s, t, r \in Z$.

Proof. Suppose that $\tilde{g}_{\delta}$ is a hybrid a-ideal of $Z$ over $U$. Since $(s * r) *(0 * t)=(s * r) *((r * t) * r) \leq s *(r * t)$, $(s * r) *(0 * t) *(s *(r * t))=0$.

By Theorem $8, \tilde{g}_{\delta}$ is a hybrid ideal of $Z$ over $U$. Thus, 


$$
\begin{aligned}
\widetilde{g}((s * r) *(0 * t)) & \supseteq \widetilde{g}(((s * r) *(0 * t)) *(s *(r * t))) \cap \widetilde{g}(s *(r * t)) \\
& =\widetilde{g}(0) \cap \widetilde{g}(s *(r * t)) \\
& \supseteq \widetilde{g}(s *(r * t)), \\
\widetilde{\delta}((s * r) *(0 * t)) & \leq \cup\{\widetilde{\delta}(((s * r) *(0 * t)) *(s *(r * t))), \widetilde{\delta}(s *(r * t))\} \\
& =\cup\{\widetilde{\delta}(0), \widetilde{\delta}(s *(r * t))\} \\
& \leq \widetilde{\delta}(s *(r * t)),
\end{aligned}
$$

for every $s, t, r \in Z$.

Theorem 10. Let $\tilde{g}_{\delta}$ be a hybrid ideal of $Z$ over $U$ satisfying the following inequality:

$$
\begin{aligned}
& \widetilde{g}(t * s) \supseteq \widetilde{g}(s *(0 * t)), \\
& \widetilde{\delta}(t * s) \leq \widetilde{\delta}(s *(0 * t)),
\end{aligned}
$$

for all $s, t \in Z$, then $\tilde{g}_{\delta}$ is a hybrid a-ideal of $Z$ over $U$.

Proof. Since $\tilde{g}_{\delta}$ is a hybrid ideal of $Z$ over $U$,

$$
\begin{aligned}
\widetilde{g}(t * s) & \supseteq \widetilde{g}(s *(0 * t)) \\
& \supseteq \widetilde{g}((s *(0 * t)) * r) \cap \tilde{g}(r) \\
& \supseteq \widetilde{g}((s * r) *(0 * t)) \cap \widetilde{g}(r), \\
\widetilde{\delta}(t * s) & \leq \widetilde{\delta}(s *(0 * t)) \\
& \leq \cup\{\widetilde{\delta}((s *(0 * t)) * r), \widetilde{\delta}(r)\} \\
& \leq \cup\{\widetilde{\delta}((s * r) *(0 * t)), \widetilde{\delta}(r)\},
\end{aligned}
$$

for all $s, t, r \in Z$. Hence, $\tilde{g}_{\delta}$ is a hybrid $a$-ideal of $Z$ over $U$.

\section{Conclusion}

In this paper, we apply the notion of hybrid structure to the ideal theory in (BCI)-algebras. In fact, we have discussed the notions of hybrid $p$-ideal, hybrid $h$-ideal, and $a$-ideal in (BCI)-algebras and investigated several related properties. Furthermore, it has been shown that every hybrid $p$-ideal (or $h$-ideal or $a$-ideal) is a hybrid ideal in a (BCI)-algebra but converse need not be true in general, and in support, examples for each case are provided. Moreover, characterizations of hybrid $p$-ideal, hybrid $h$-ideal, and hybrid $a$-ideal in (BCI)-algebras are discussed.

\section{Data Availability}

No data were used to support this study.

\section{Conflicts of Interest}

The authors declare that they have no conflicts of interest.

\section{Acknowledgments}

This work was supported by the Taif University Researchers Supporting Project (TURSP-2020/246), Taif University, Taif, Saudi Arabia.

\section{References}

[1] Y. Imai and K. Iséki, "On axiom systems of propositional calculi, XIV," Proceedings of the Japan Academy, vol. 42, no. 1, pp. 19-22, 1966.

[2] K. Iséki, "An algebra related with a propositional calculus," Proceedings of the Japan Academy, vol. 42, pp. 26-29, 1966.

[3] Y. B. Jun and C. H. Park, "Applications of soft sets in ideal theory of BCK/BCI-algebras," Information Sciences, vol. 178, pp. 2466-2475, 2008.

[4] H. M. Khalid and B. Ahmad, "Fuzzy $h$-ideals in BCI-algebras," Fuzzy Sets and Systems, vol. 101, no. 1, pp. 153-158, 1999.

[5] Y. L. Liu, J. Meng, and X. H. Zhang, " $q$-ideals and a-ideals in BCI-Algebras," Southeast Asian Bulletin of Mathematics, vol. 24, no. 2, pp. 243-253, 2000.

[6] G. Muhiuddin, D. Al-Kadi, A. Mahboob, and K. P. Shum, "New types of bipolar fuzzy ideals of BCK-algebras," International Journal of Analysis and Applications, vol. 18, no. 5, pp. 859-875, 2020.

[7] L. A. Zadeh, "Fuzzy sets," Information and Control, vol. 8, no. 3, pp. 338-353, 1965.

[8] D. Molodtsov, "Soft set theory-First results," Computers \& Mathematics with Applications, vol. 37, no. 4-5, pp. 19-31, 1999.

[9] D. A. Molodtsov, "The description of a dependence with the help of soft sets," Journal of Computer and Systems Sciences International, vol. 40, no. 6, pp. 977-984, 2001.

[10] D. A. Molodtsov, The Theory of Soft Sets, URSS Publishers, Moscow, Russia, in Russian, 2004.

[11] Y. B. Jun, "Soft BCK/BCI-algebras," Computers \& Mathematics with Applications, vol. 56, no. 5, pp. 1408-1413, 2008.

[12] Y. B. Jun, K. J. Lee, and C. H. Park, "Soft set theory applied to ideals in $d$-algebras," Computers and Mathematics with Applications, vol. 57, pp. 367-378, 2009.

[13] H. Aktaş and N. Çağman, "Soft sets and soft groups," Information Sciences, vol. 177, pp. 2726-2735, 2007.

[14] Y. B. Jun, K. J. Lee, and A. Khan, "Soft ordered semigroups," Mlq, vol. 56, no. 1, pp. 42-50, 2010.

[15] U. Acar, F. Koyuncu, and B. Tanay, "Soft sets and soft rings," Computers \& Mathematics with Applications, vol. 59, no. 11, pp. 3458-3463, 2010.

[16] F. Feng, Y. B. Jun, and X. Zhao, "Soft semirings," Computers \& Mathematics with Applications, vol. 56, no. 10, pp. 2621-2628, 2008.

[17] N. Çağman, F. Çitak, and S. Enginoğlu, "Soft set theory and uni-int decision making," European Journal of Operational Research, vol. 207, pp. 848-855, 2010.

[18] P. K. Maji, A. R. Roy, and R. Biswas, "An application of soft sets in a decision making problem," Computers and Mathematics with Applications, vol. 44, no. 8-9, pp. 1077-1083, 2002. 
[19] G. Muhiuddin and S. Aldhafeeri, "Characteristic fuzzy sets and conditional fuzzy subalgebras," Journal of Computational Analysis and Applications, vol. 25, no. 8, pp. 1398-1409, 2018.

[20] G. Muhiuddin and A. M. Al-roqi, "Classifications of $(\alpha, \beta)$-fuzzy ideals in BCK/BCI-algebras," Journal of Computational Analysis, vol. 7, no. 6, pp. 75-82, 2016.

[21] G. Muhiuddin and A. M. Al-roqi, "Subalgebras of BCK/BCIalgebras based on $(\alpha, \beta)$-type fuzzy sets," Journal of Computational Analysis and Applications, vol. 18, no. 6, pp. 1057-1064, 2015.

[22] G. Muhiuddin, M. E. A. Elnair, A. Mahboob, and K. P. Shum, "Energetic sets and $(\epsilon, \epsilon V q)$-permeable values in BCK/BCIalgebras," Journal of Interdisciplinary Mathematics, vol. 22, no. 8, p. 1223, 2019.

[23] G. Muhiuddin and K. P. Shum, "New types of $(\alpha, \beta)$-fuzzy subalgebrasof BCK/BCI-algebras," International Journal of Mathematics and Computer Science, vol. 14, no. 2, pp. 449464, 2019.

[24] M. Farooq, T. Mahmood, A. Khan, M. Izhar, and B. Davvaz, "Fuzzy hyperideals of hyperquantales," Journal of Intelligent \& Fuzzy Systems, vol. 36, no. 6, pp. 5605-5615, 2019.

[25] K. Hayat and T. Mahmood, "Some applications of bipolar soft set: characterizations of two isomorphic hemi-rings via BSI- $h$ ideals," British Journal of Mathematics \& Computer Science, vol. 13, no. 2, pp. 1-21, 2016.

[26] T. Mahmood, "New types of interval valued fuzzy $h$-ideals of hemirings," World Applied Science Journal, vol. 18, pp. 1883-1889, 2012.

[27] T. Mahmood, M. I. Ali, and A. Hussain, "Generalized roughness in fuzzy filters and fuzzy ideals with thresholds in ordered semigroups," Computational and Applied Mathematics, vol. 37, no. 4, pp. 5013-5033, 2018.

[28] T. Mahmood and A. Ejaz, "On bipolar valued fuzzy $k$-ideals in hemirings,” The Nucleus, vol. 52, no. 3, pp. 115-122, 2015.

[29] T. Mahmood and K. Hayat, "Applications of soft intersection set in B-algebras via SI-B-ideals," International Journal of Algebra and Statistics, vol. 4, no. 2, pp. 57-62, 2015.

[30] T. Mahmood and K. Hayat, "Characterizations of hemi-rings by their bipolar-valued fuzzy h-ideals," Information Sciences Letter, vol. 4, no. 2, pp. 51-59, 2015.

[31] T. Mahmood and M. Shabir, "Characterizations of $h$-hemiregular and $h$-semisimple Hemirings by interval valued fuzzy h-ideals," World Applied Science Journal, vol. 17, no. 12, pp. 1821-1827, 2012.

[32] G. Muhiuddin, A. Mahboob, and N. Mohammad Khan, "A new type of fuzzy semiprime subsets in ordered semigroups," Journal of Intelligent \& Fuzzy Systems, vol. 37, no. 3, pp. 4195-4204, 2019.

[33] G. Muhiuddin and A. Mahboob, "Int-soft ideals over the soft sets in ordered semigroups," AIMS Mathematics, vol. 5, no. 3, pp. 2412-2423, 2020.

[34] M. Shabir, S. Liaquat, and S. Bashir, "Regular and intraregular semirings in terms of bipolar fuzzy ideals," Journal of Computational and Applied Mathematics, vol. 38, no. 197, 2019.

[35] Y. B. Jun, S.-Z. Song, and G. Muhiuddin, "Hybrid structures and applications," Annals of Communications in Mathematics, vol. 1, no. 1, pp. 11-25, 2018.

[36] S. Anis, M. Khan, and Y. B. Jun, "Hybrid ideals in semigroups," Cogent Mathematics, vol. 4, no. 1, Article ID 1352117, 2017.

[37] B. Elavarasan and Y. B. Jun, "Regularity of semigroups in terms of hybrid ideals and hybrid bi-ideals," Kragujevac Journal of Mathematics, vol. 46, no. 6, pp. 857-864, 2022.
[38] B. Elavarasan, K. Porselvi, and Y. B. Jun, "Hybrid generalized bi-ideals in semigroups," International Journal of Mathematics and Computer Science, vol. 14, no. 3, pp. 601-612, 2019.

[39] K.-T. Kang, S.-Z. Song, E. H. Roh, and Y. B. Jun, "Hybrid ideals of BCK/BCI-algebras," Axioms, vol. 9, no. 3, p. 85, 2020.

[40] G. Muhiuddin, D. Al-Kadi, and A. Mahboob, "Ideal theory of BCK/BCI-algebras based on hybrid structures," Journal of Mathematics and Computer Science, vol. 23, no. 2, pp. 136144, 2021.

[41] Y. B. Jun and J. Meng, "Fuzzy p-ideals in BCI-algebras," Journal of the Mathematical Society of Japan, vol. 40, pp. 271-282, 1994.

[42] X. H. Zhang, J. Hao, and S. A. Bhatti, "On p-ideals of a BCI-algebra," Punjab University Journal of Mathematics, vol. 27, pp. 121-128, 1994.

[43] Y. Huang, BCI-Algebra, Science Press, Beijing, China, 2006.

[44] K. Iséki, "On BCI-algebras," Mathematics Seminar Notes, vol. 8, pp. 125-130, Kobe University, Kobe, China, 1980.

[45] J. Meng and Y. B. Jun, BCK-Algebras, Kyungmoon Sa Co. Seoul, Seoul, Korea, 1994. 